\title{
Intelligent Observer-Based Controller Design for Nonlinear Type-1 Diabetes Model via Adaptive Neural Network Method
}

\author{
Elham Rahimi Khoygani a,1,* Mohammad Reza Rahimi Khoygani ${ }^{\text {a,b,2, Reza Ghasemi }}{ }^{\text {b,3 }}$ \\ ${ }^{a}$ Department of Control Engineering, Islamic Azad University, Tehran, Iran \\ ${ }^{\mathrm{b}}$ Electrical Engineering department. university of qom, Qom, Iran \\ ${ }^{1}$ Elham.rahimikh@gmail.com; ${ }^{2}$ Mrrahimikh@gmail.com; ${ }^{3}$ R.Ghasemi@qom.ac.ir \\ * Corresponding Author
}

ARTICLE INFO

\section{Article History}

Received 05 September 2021

Revised 24 September 2021

Accepted 26 September 2021

\section{Keywords}

Type-1 diabetes;

Nonlinear controller;

Lyapunov stability;

Neural network;

Adaptive method;

Artificial intelligence

\section{ABSTRACT}

Diabetes is an increasing health problem all around the world, particularly Type 1 diabetes (T1D), people with T1D require precise glycemic control, due to a shortage of insulin production. This paper introduces a new adaptive neural observer-based controller for a class of nonlinear T1D systems. A solution is proposed to guarantees practical tracking of a desired glucose concentration by a new adaptive neural observer-based control strategy. One of the intelligence procedures is the network under online learning that the mentioned controller is learned by a backpropagation algorithm. This network is a significant class of feed-forward artificial neural networks that maps a set of inputs into a set of proper outputs. Guarantee stability of observer and controller by Lyapunov direct and training online are the merit of the method. Also, despite the presence of internal and external uncertainties, the multilayer perceptron neural observer-based controller is robust. The performance of the proposed method is hopeful based on the results.

This is an open access article under the CC-BY-SA license.

\section{Introduction}

The Insufficient generation or shortage counteraction to insulin is caused to create this metabolic illness [1] which one of the most important chronic diseases is diabetes. More than a few hundred million people suffer from diabetes worldwide, and it is predicted that this number will double in the next 30 years [2]. In later decades, significant growth in diabetes outbreaks has shown stability and latency, while several million people worldwide get involved in diabetes [11]. According to the International Diabetes Federation, 425 million people in 2017 had type I diabetes (T1D), and as a perspective by 2045, the number could reach up to 629 million, which is a worldwide growth of $48 \%$ in only 28 years [12]. As per research, 327 million patients worldwide are an average age of 20-64 years; this is a $72 \%$ increase over the past 65 years. The worldwide growth from 2017-2045 is enormous; in North America and the Caribbean, a projection of $35 \%$ could raise the cases of T1D that can increase rapidly to type- 2 diabetes. In Europe is predicted an increase of $62 \%$; in the meantime, in the South-Central Americas is expected a $16 \%$ growth in the population with T1D. The lowest estimated growth is related to the Western Pacific area with $15 \%$. According to the report, further growth is related to southeast 
Asia, Africa, and the Middle East that go from $72 \%$ up to $156 \%$, which shows warning state that requires political strategies and more investigating. Diabetes-related side problems are a pandemic around the world that has high economic, medical, and social costs [4]. Besides, it is estimated that about $9 \%$ of all deaths worldwide have occurred from diabetes [3]. People's life all around the world is affected by diabetes regardless of race, sex, nationality, or social status despite diabetes being an immunological disease [10]. There are lots of natural feedback loops inside the human body, which are in charge of maintaining homeostasis. The defect or failure of one of these loops can make severe illnesses with short or long-term complications. Diabetes can lead to problems like brain hurt, nerve hurt, mutilation, and eventually death if the blood glucose level is not properly controlled. Insulin is a hormone, which regulates glucose uptake by the body cells.

There are two types of diabetes, type I diabetes or insulin-dependent and type 2 diabetes or non-insulin-dependent [5]. It is predicted that the number of patients under the age of 20 with T1D or type-2 reaches its peak until 2045 [8][9]. In type 1 diabetes, pancreatic beta cells are destroyed so the pancreas does not secrete insulin. Thus the effect of glucagon which causes to elevate the concentration of glucose in the blood cannot be suppressed by insulin. In this case, the patients need exogenous insulin injections to process and regulate their glucose concentration to a normal level. There are two situations depending on glucose concentration, namely, hyperglycemia and hypoglycemia. Moreover, type-1 diabetes (T1D) or Mellitus diabetes which the production of pancreatic b-cells decays at a rate proportional to leukocyte cell growth [7]. When lymphocytes T-cells diagnose pancreatic b-cells as antigens, diabetes is created. When b-cells destroy increasingly, causes an absolute lack of insulin production and dysregulation of glucose metabolism. If the glucose concentration is remarkably higher than the normal level, hyperglycemia occurs [6]. Hypoglycemia occurs when the glucose concentration is less than normal [6]. Both hypoglycemia and hyperglycemia can impress the patient's health and life. It is essential for a diabetic person to perform manually the procedures of blood glucose regulation. AS the predictions are globally enormous in terms of how T1D is increasing over time, future health will be improved by robust strategies based on mathematical modeling. Also, due to limited understanding of the pancreas' organogenesis and restricted access to preliminary tissues, the advancement of novel treatment options is stopped. Therefore, the existence of a system that controls automatically the blood glucose level of a diabetic will be one of the most useful devices in community health. This system permits the patient to participate in normal daily activities with risk mitigation of long-term side effects.

The inference of the consequences of interactions is done using the analysis of models through computational and applied mathematical methods [13][14][15][16]. Connections between network structure and behavior are created by providing insights into why a system behaves [20]. In addition, with the help of mathematical models, the cause-and-effect process can be formalized and related to biological observations [17]. In control engineering particularly in nonlinear system control, they have special importance [33] [34]. There are several methods for delivering insulin using the feedback controller, such as classical methods such as PID [21][22] and pole placement [23], which need a linear model for design, also model predictive control [24][25]. Numerous studies have been performed in recent years to intelligently control blood glucose. In the study [26], for reduction of the time of descending blood glucose, a PID controller based on neural networks is suggested. In [27], for minimizing the time that lasts for blood glucose to return to its basic level, several parameters of the Hammerstein controller were optimized. In [31], an online glucose-insulin identification using the Recurrent High Order Neural Network is proposed. A smart artificial pancreas for treating Type 1 Diabetes Mellitus in the elderly is presented In work [32]. Moreover, for use non-model-based controllers like fuzzy controllers and neural networks have been attempted [28][29]. 
One of the artificial intelligence techniques that can be used to develop the performance of devices and industrial processes is the artificial neural network. Approximation of unknown nonlinear functions has been done by general approximation capabilities, conformity, and learning capabilities. One of the most efficient tools for designing a nonlinear observer system is them [18]. Using the fact that a vast range of nonlinear functions with any demanded degree of accuracy under specific conditions networks can estimate, lots of research has been conducted in the proposed observer [19]. Meanwhile, to classify patterns, forecasting, diagnosis, and approximation, multi-layer perceptron neural networks are used. Indeed, these implementations are because of their capacity for nonlinear functions' approximation [19]. Multilayer networks abilities have quickly increased in order to learning and modeling in recent years. This study presents and describes a mathematical model of the disease that analysis of the complex cellular relation between pancreatic cells. Then, introduce a new adaptive neural observer-based controller for a class of nonlinear diabetes models. After that, the stability of the observer and controller are Guaranteed by the Lyapunov direct method. Despite the presence of external and internal uncertainties, the Multilayer perceptron neural observer-based controller is robust. Finally, a nonlinear T1D model is simulated by MATLAB in order to show the performance of the proposed method.

The paper is organized as follows: A brief overview of the mathematical model is presented in Section 2. the adaptive neural observer-based controller is introduced in Section 3. To proof the advantages of the introduced method, numerical simulation is done in Section 4. Finally, the conclusions are provided in Section 5.

\section{Mathematical Model of T1D}

According to [30], resting macrophages have a constant supply rate $a$ with a natural death rate $c$. The dynamics of each cell population set are as equation (1):

$$
\dot{M}(t)=a+(b+K) M_{A}(t)-c M(t)-g M(t) A(t)
$$

where $M$ is the interaction of resting macrophages, $M_{A}$ is activated macrophages, and antigen cells shows $A$. Increase due to the recruitment of activated macrophages with a maximum rate $b+k$, while $g$ is the rate at which resting macrophages become active due to interaction with antigenic cells. Activated macrophages have a supply rate g due to interaction between resting macrophages and antigenic cells, and a natural death rate $k$.

$$
\dot{M}_{A}(t)=g M(t) A(t)-k M_{A}(t)
$$

Antigen cells increase due to the release of both antigenic peptides by activated macrophages and $\beta$-cell antigenic peptides by dead $\beta$-cells due to the interaction between b-cells and T-cells with a rate $l$ and $q$, respectively. $m$ is the rate at which antigenic cells are cleared from their population.

$$
\dot{A}(t)=l M_{A}(t)+q B(t) T(t)-m A(t)
$$

Autolytic T-cells have a constant supply rate $s_{T}$ and a natural death rate $\mu_{T}$ with proliferation rate $s$ due to a profile of cytokines and chemokines, induced by activated macrophages.

$$
\dot{T}(t)=s_{T}+s M_{A}(t) T(t)-\mu_{T} T(t)
$$

$\beta$-Cells have a constant supply rate $s_{B}$ and a natural death rate $\mu_{B}$, while $q$ is the depletion of the $\beta$-cell population due to interactions between $\beta$-cells and T-cells. The description of each parameter and its estimation. Autolytic T cells (T), and cells (B) is modelled by the following system of equations.

$$
\dot{B}(t)=s_{B}-q B(t) T(t)-\mu_{B} B(t) .
$$


Now these equations may be represented into state space form by considering the state variables as following:

$$
x_{1}=M \quad, \quad x_{2}=M_{A} \quad, \quad x_{3}=A \quad, \quad x_{4}=T, \quad x_{5}=B
$$

The biological implication due to a population set of resting macrophages $\left(x_{1}\right)$ that label a population set of $\beta$-cells $\left(x_{5}\right)$ as antigens $\left(x_{3}\right)$, thereby $x_{1}$ becomes activated macrophages $\left(x_{2}\right)$ and directly responsible for autolytic T-cell $\left(x_{4}\right)$ stimulation that their primary function is to trigger the lymphocytes' cytotoxic T-cells response. That is, the interaction of the population sets of resting macrophages, activated macrophages, antigens, autolytic T-cells, and $\beta$-cells. In accordance with the aforementioned, system (6) can be expressed in closed-loop control form as follows:

$$
\left\{\begin{array}{l}
\dot{x}(t)=F_{d}(x)+G_{d} u(t) \\
y(t)=C x(t)
\end{array}\right.
$$

where $F_{d}, G_{d}$, and $u$ are cosidered as follows:

$$
\left\{\begin{array}{c}
\dot{x}(t)=\left[\dot{x}_{1}, \dot{x}_{2}, \dot{x}_{3}, \dot{x}_{4}, \dot{x}_{5}\right]^{T} \\
F_{d}=\left[f_{d 1}, f_{d 2}, f_{d 3}, f_{d 4}, f_{d 5}\right]^{T} \\
u(t)=\left[u_{1}, u_{2}, u_{3}\right]^{T} \\
G_{d}=\left[\begin{array}{lll}
0 & 0 & 0 \\
1 & 0 & 0 \\
0 & 1 & 0 \\
0 & 0 & 1 \\
0 & 0 & 0
\end{array}\right]
\end{array}\right.
$$

Also

$$
\left\{\begin{array}{l}
f_{d 1}=a+(b+k) x_{2}(t)-c x_{1}(t)-g x_{1}(t) x_{3}(t) \\
f_{d 2}=g x_{1} x_{3}(t)-k x_{2}(t) \\
f_{d 3}=l x_{2}(t)-m x_{3}(t)+q x_{4}(t) x_{5}(t) \\
f_{d 4}=s_{T}+s x_{2}(t) x 4(t)-\mu_{T} x_{4}(t) \\
f_{d 5}=s_{B}-q x_{4}(t) x_{5}(t)-\mu_{B} x_{5}(t)
\end{array}\right.
$$

This paper has been proposed three control inputs for the variables related to activated macrophages, b-cells presented as antigens, and autolytic T-cells. The control theory for improving the immunological response against T1D is presented. Therefore, inhibiting the $x_{2}$ responses would contribute to a decreasing $x_{5}$ labeled as $x_{3}$, and as a consequence, it will not be eliminated. Hence, this method must be controlling the populations of these undesired cells.

\section{Control design}

In this section, an effective intelligent nonlinear observer based controller is proposed for Blood Glucose of T1D model via adaptive neural network.

\subsection{Neural observer}

Nonlinear neural observer under a conventional backpropagation are followed to improve performance. A three-layer structure of the multilayer perceptron network is considered as $G_{o b}(x, u)$ which shows in Fig. 1. By using a multilayer perceptron for some sufficiently large number of hidden layer neurons, there exist weights and thresholds so that a continuous function $G_{o b}(x, u)$ can be represented as:

$$
G_{o b}(x(t), u(t))=W^{T}(t) \sigma\left(V^{T}(t) \bar{x}(t)\right)+\varepsilon(t)
$$






Fig. 1. Structure of the multilayer perceptron neural network

in above, $\varepsilon \in R^{n}$ is the bounded approximation error of the neural network, $V(t) \in R^{q \times q}$ is weight matrix of the hidden layer, and $W(t) \in R^{n \times q}$ is weight matrix of the output layer that are considered as follows:

$$
W(t)=\left[\begin{array}{cccc}
W_{11} & W_{12} & \ldots & W_{1 q} \\
W_{21} & W_{22} & \ldots & W_{2 q} \\
\ldots & \ldots & \ldots & \ldots \\
W_{n 1} & W_{n 2} & \ldots & W_{n q}
\end{array}\right] \quad, \quad V(t)=\left[\begin{array}{cccc}
V_{11} & V_{12} & \ldots & V_{1 q} \\
V_{21} & V_{22} & \ldots & V_{2 q} \\
\ldots & \ldots & \ldots & \ldots \\
V_{q 1} & V_{q 2} & \ldots & V_{q q}
\end{array}\right]
$$

it is assumed where there exist an upper bound for the fixed ideal weights $W(t)$ and $V(t)$ :

$$
\|W\| \leq W_{M} \quad, \quad\|V\| \leq V_{M}
$$

where $\sigma\left(V^{T} \bar{x}\right) \in R^{n+m}$ is transfer function of the hidden neurons.

In the output and hidden layers, an activation function was considered in order to describing the nonlinear characteristics of the network. The linear functions often was used for output layer, the linear threshold, sigmoidal, hard limit, gaussian, and hyperbolic tangent usually were used for hidden layer, In this work, a tangent hyperbolic function is used as the activation function. Also, the upper bound for $\sigma(V \bar{x})$ is assumed as follows:

$$
\|\sigma(V \bar{x})\| \leq \sigma_{M}
$$

Due to the mentioned definitions, the proposed wavelet network able to estimate any non-linear function such as $f \in L^{2}\left(R^{q}\right)$ (continuous or discontinuous, finite-energy) by an arbitrary precision. Fig. 1 depicts a structure of the the MLP network. The nonlinear model in the presence of disturbance is considered in Equation (8). Therefore, equation (13) is obtained from subtracting and adding phrase $A x(t)$ into (7), then

$$
\begin{aligned}
& \dot{x}(t)=A x(t)+G_{o b}[x(t), u(t)] \\
& y(t)=C x(t)
\end{aligned}
$$

in above equation, the known output matrix is $C \in R^{p \times n}, A$ is optional matrix which the eigenvalue of it has negative real part (Hurwitz) and both matrixes $M_{b}, C$ are observable. $G_{o b}(x(t)$, $u(t))$ is a the unknown nonlinear function that includes uncertain and disturbance terms, such that

$$
G_{o b}(x(t), u(t))=F_{d}(x)+G_{d}(x) U(t)-A x(t)
$$


from the Luenberger observer [18], equation of the nonlinear system (16) is assumed as:

$$
\begin{aligned}
& \dot{\hat{x}}(t)=A \hat{x}+\hat{G}_{o b}(\hat{x}(t), u(t))+\Gamma e(t) \\
& \hat{y}(t)=C \hat{x}(t)
\end{aligned}
$$

where $\Gamma \in \mathbb{R}^{n}$ is the observer gain which it must be chosen such that $A-\Gamma C$ becomes a Hurwitz matrix, and $\hat{x}(t)$ is state of the observer. To approximate $G_{o b}(x(t), u(t))$ a MLP neural net is considered that it has enough large number of hidden layer neurons in order to estimate the unknown nonlinear function (15).

Fig. 2 shows the structure of the multilayer neural adaptive observer. Therefore, unit matrix $I$ is considered as the first layer weight $V=I$.

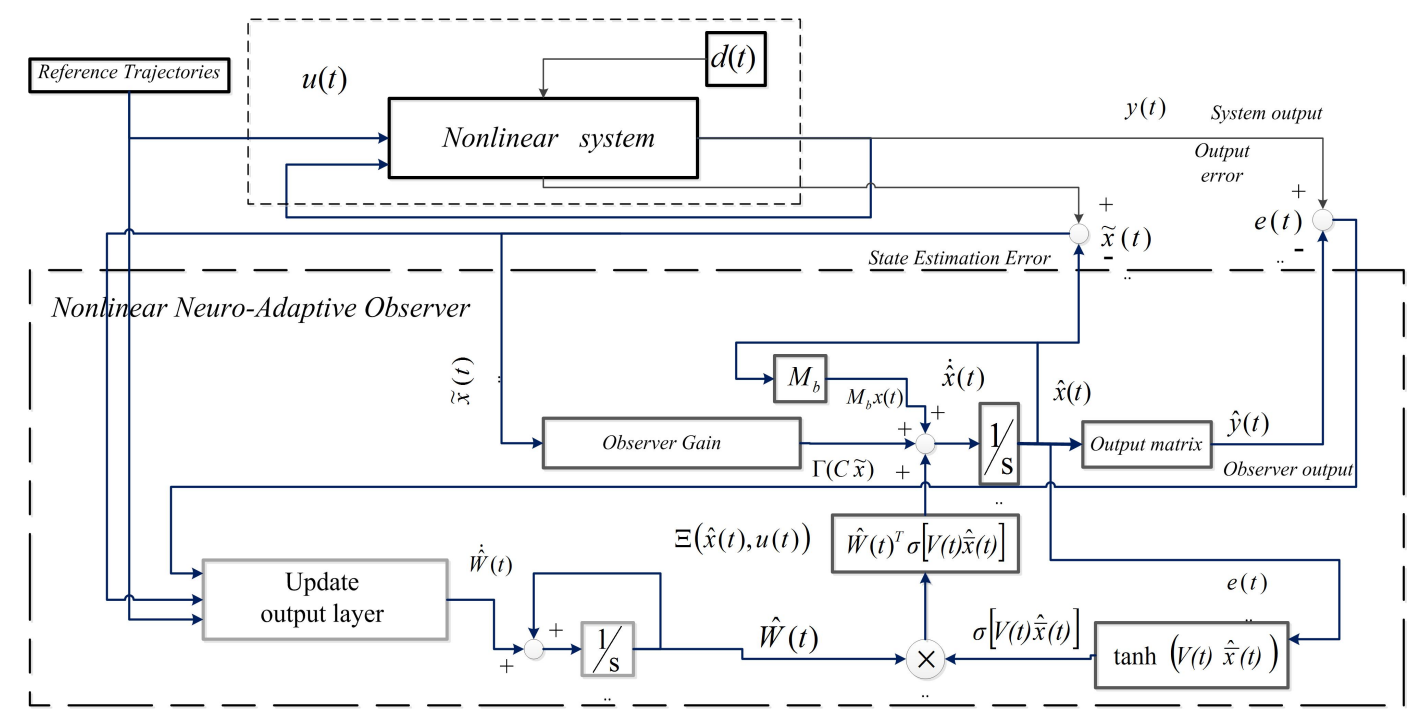

Fig. 2. Structure of the neural observer

Hence, the proposed nonlinear observer can be written as follows:

$$
\hat{G}_{o b}(\hat{x}(t), u(t))=\hat{W}^{T} \sigma(\hat{\bar{x}}(t))+\varepsilon(t)=\hat{W}^{T} \tanh (\hat{x}(t), u(t))+\varepsilon(t)
$$

the weight error is assumed as:

$$
\tilde{W}=W(t)-\hat{W}(t)
$$

by using output error $e(t)=y(t)-\hat{y}(t)$ and (15) into (17), the nonlinear observer is given as follows:

$$
\begin{aligned}
\dot{\hat{x}}(t)= & A \hat{x}(t)+\hat{W}^{T}(t) \tanh (\hat{x}(t), u(t)) \\
& +\Gamma(y(t)-C \hat{x}(t))
\end{aligned}
$$

according to (15), (18) and $\dot{\tilde{x}}(t)=\dot{x}(t)-\dot{\hat{x}}(t)$ can be writen:

$$
\begin{array}{rl}
\dot{\tilde{x}}(t)=A & x(t)-A \hat{x}(t)+W^{T}(t) \tanh (x(t), u(t)) \\
& -\hat{W}^{T}(t) \tanh (\hat{x}(t), u(t))+\Gamma(C \tilde{x}(t))+\varepsilon(t)
\end{array}
$$

using the estimation error $\tilde{x}=x(t)-\hat{x}(t)$ into (21):

$$
\dot{\tilde{x}}(t)=A_{b} \tilde{x}(t)+\tilde{W}^{T}(t) \tanh (\tilde{x}(t), u(t))+\Delta(t)
$$


in above equation, $A_{b}$ represents $A-\Gamma C$ and:

$$
\Delta(t)=W(t)[\tanh (x, u)-\tanh (\hat{x}, u)]+\varepsilon(t)
$$

is a bounded disturbance term [102] that $\|\varepsilon(t)\| \leq \varepsilon_{M}$, therefore, can be assumed:

$$
\|\Delta(t)\| \leq K_{\Delta} .
$$

when the neural network structure is well known, a suitable learning rule should be appointed to educate the network. In addition, the back propagation algorithm are presented to improve neural observer performance for state estimation of the fuel cell system. Conventional back propagation is a popular algorithm [19]. The weight adjustment mechanism is given as follows:

$$
\dot{\hat{W}}(t)=-\eta \frac{\partial \varpi(t)}{\partial \hat{W}(t)}-\rho\|e\| \hat{W}(t)
$$

where $\eta>0$ and $\varpi=0.5\left(e^{T} e\right)$ are the learning rate and the objective function. By using $e=$ $y-C \hat{x}$, the $\partial \varpi / \partial \hat{W}$ is computed according to;

$$
\frac{\partial \varpi}{\partial \hat{W}}=\frac{\partial \varpi}{\partial e} \frac{\partial e}{\partial \hat{x}} \frac{\partial \hat{x}}{\partial \hat{W}}=-e^{T} C \frac{\partial \hat{x}}{\partial \hat{W}}
$$

where $(\partial \varpi / \partial \hat{W})$ a set of nonlinear dynamical system that should be used for solving the gradient $\partial \varpi / \partial \hat{W}$. Hence, this makes a complex observer and the real-time performance becomes very hard. The $(\partial \hat{x} / \partial \hat{W}) \approx-M^{-1}(\partial \hat{\Xi} / \partial \hat{W})$ is static approximation that is used to solve this problem, then

$$
\frac{\partial \varpi}{\partial \hat{W}}=e^{T} C M^{-1} \frac{\partial \hat{\Xi}}{\partial \hat{W}}
$$

now by using (25) and $e=C \tilde{x}$, the learning rule (28) is obtained:

$$
\dot{\hat{W}}(t)=-\eta\left(\tilde{x}^{T} C^{T} C M^{-1}\right)^{T}(\sigma(\hat{\bar{x}}))^{T}-\rho\|e\| \hat{W}(t)
$$

where $\rho$ is a small positive number, using the weight error is changed as:

$$
\dot{\tilde{W}}(t)=\eta\left(\tilde{x}^{T} C^{T} C M^{-1}\right)^{T}(\sigma(\hat{\bar{x}}))^{T}+\rho\|C \tilde{x}\| \hat{W}(t)
$$

Theorem 1: Consider the fractional order nonlinear uncertain system with unknown dynamics (13) and nonlinear neuro-adaptive observer (20). If the weights of the multilayer perceptron were updated via adaptive law (27), then $\tilde{x}, \tilde{w}$, and e are guaranteed as uniformly ultimately bounded .

Then, to determine the conditions for each control input, we consider the following Lyapunov candidate function:

$$
L_{f}(t)=\frac{1}{2} \tilde{x}^{T} B_{l} \tilde{x}+\frac{1}{2} \operatorname{tr}\left(\tilde{w}^{T} \tilde{w}\right)
$$

where the $B_{l}=B_{l}^{T}$ and $Q$ are positive-definite matrix and the $M_{b}$ is the Hurwitz matrix satisfying:

$$
A_{b}^{T} B_{l}+B_{l} A_{b}=-Q
$$

the time derivative of function (28) can be written by:

$$
\dot{L}_{f}=\frac{1}{2} \tilde{x}^{T} B_{l} \dot{\tilde{x}}+\frac{1}{2} \dot{\tilde{x}}^{T} B_{l} \tilde{x}+\operatorname{tr}\left(\tilde{w}^{T} \dot{\tilde{w}}\right)
$$


by substituting equations (20), (27), and $\dot{\tilde{w}}=-\dot{\hat{w}}$ into (35), it gives:

$$
\begin{aligned}
\dot{L}_{f} \leq & -\frac{1}{2} \tilde{x}^{T} Q \tilde{x}+\tilde{x}^{T} B_{l}\left(\tilde{w}^{T} \mho(\hat{\bar{x}}, u)+\Delta(t)\right) \\
& +\operatorname{tr}\left(\tilde{w}^{T} \eta_{b} \mho(\hat{\bar{x}})\left(e^{T} C A_{b}^{-1}\right)+\tilde{w}^{T} \rho_{b}\|e\| \hat{w}\right)
\end{aligned}
$$

Also, $\operatorname{tr}(B)$ denotes the trace of $B$, which leads to the inequality $\operatorname{tr}\left[A B^{T}\right]=B^{T} A$. Based on the inequality, (24), and (34) can be written as:

$$
\left\{\begin{array}{l}
\operatorname{tr}\left(\tilde{w}^{T}(w-\tilde{w})\right) \leq w_{M}\|\tilde{w}\|-\|\tilde{w}\|^{2} \\
\operatorname{tr}\left(\tilde{w}^{T} \mho(\overline{\bar{x}}, u) \tilde{x} \alpha_{1}\right) \leq \mho_{M}\|\tilde{w}\|\|\tilde{x}\|\left\|\alpha_{1}\right\|
\end{array}\right.
$$

according to the equations (11), (12), (22), (29) and (32) can be rewritten as:

$$
\begin{aligned}
\dot{L}_{f} & \leq-\frac{1}{2} \lambda_{\min }(Q)\|\tilde{x}\|^{2}+\|\tilde{x}\|\left\|B_{l}\right\|\left(\|\tilde{w}\| \mho_{M}+K_{\Delta}\right) \\
& +\mho_{M}\|\tilde{w}\|\|\tilde{x}\|\left\|\eta_{b} e^{T} C A_{b}^{-1}\right\|+\left(w_{M}\|\tilde{w}\|-\|\tilde{w}\|^{2}\right) \rho_{b} C\|\tilde{x}\|
\end{aligned}
$$

so that:

$$
\dot{L}_{f} \leq-\left(Z_{1}\|\tilde{x}\|-K_{\Delta}\left\|B_{l}\right\|\right)\|\tilde{x}\|-\left(\left\|B_{l} C\right\|\|\tilde{x}\|-Z_{2}\right)\|\tilde{W}\|^{2}
$$

where terms $Z_{1}$ and $Z_{2}$ are defined by

$$
\left\{\begin{array}{l}
Z_{1}=0.5\left(\lambda_{\min }(Q)-\mho_{M}\left\|B_{l}\right\|-\mho_{M}\left\|B_{l} C\right\|\right) \\
Z_{2}=0.5\left(\mho_{M}\left\|B_{l}\right\|+\mho_{M}\left\|\eta_{b} e^{T} C A_{b}^{-1}\right\|+W_{M}\left\|B_{l} C\right\|\right)
\end{array}\right.
$$

To ensure negative definiteness of $\dot{L}_{f}$, is negative long the following conditions:

$$
\|\tilde{x}\|>\max \left(s_{1}, s_{2}\right)
$$

where

$$
s_{1}=\frac{K_{\Delta}\|B\|}{Z_{1}} \quad, \quad s_{2}=\frac{Z_{2}}{\|B C\|}
$$

it is obvious that $\dot{L}_{f}$ is negative definite. Therefore, the observer error $\tilde{x}(t)$ is uniformly ultimately bounded.

Remark 1 According to Theorem 1, all the estimation errors $\tilde{x}_{1}, \ldots, \tilde{x}_{5}$ are small and uniformly ultimately bounded. Hence, this here can be assumed the upper bound for the estimation errors as $\left(\tilde{x}_{1}, \ldots, \tilde{x}_{5}\right) \leq\left(X_{M 1}, \ldots, X_{M 5}\right)$.

\subsection{Controller}

This section shows three control inputs for the variables related to activated macrophages, b-cells presented as antigens and autolytic T-cells. The structure T1D and neural observer-based controller is displayed in Fig. 3. In accordance with the aforementioned, the closed-loop control without observer for system (7) can be expressed as follows:

$$
\begin{aligned}
& u_{1}(t)=-g x_{1} x_{3} \\
& u_{2}(t)=-l x_{2}-q x_{5} x_{4} \\
& u_{3}(t)=-s x_{2} x_{4}
\end{aligned}
$$


and closed-loop observer based controller is

$$
\left\{\begin{array}{l}
u_{o 1}(t)=-g \hat{x}_{1} \hat{x}_{3} \\
u_{o 2}(t)=-l \hat{x}_{2}-q \hat{x}_{5} \hat{x}_{4} \\
u_{o 3}(t)=-s \hat{x}_{2} \hat{x}_{4}
\end{array}\right.
$$

where $u_{1}, u_{2}$, and $u_{3}$ are control inputs that in a biological sense have the objective of preserving b-cell population in pancreas islets. According to Remark 1, the difference between the inputs controller under the observer and the input without observer are defined by the delta symbol $\left(\delta_{1,2,3}=u_{1,2,3}-u_{o 1,2,3}\right)$ that are a small constant.

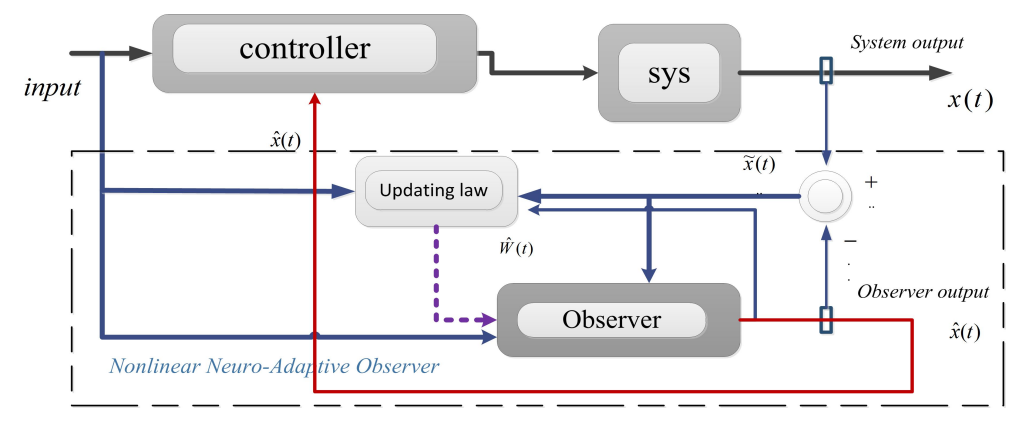

Fig. 3. The structure T1D and neural observer-based controller

Analyse stability: The following Lyapunov candidate function is defined in order to determine the conditions for each control input:

$$
L_{c}=\frac{1}{2} \sum_{i=1}^{5} \beta_{i} x_{i}^{2}
$$

where the derivative of Lyapunov function is given as follows:

$$
\dot{L}_{c}=\beta_{1} x_{1} \dot{x}_{1}+\beta_{2} x_{2} \dot{x}_{2}+\beta_{3} x_{3} \dot{x}_{3}+\beta_{4} x_{4} \dot{x}_{4}+\beta_{5} x_{5} \dot{x}_{5}
$$

and using (7) into the derivative of (41), is defined by

$$
\begin{aligned}
\dot{L}_{c}= & \beta_{1} x_{1}\left(a+(b+k) x_{2}-c x_{1}-g x_{1} x_{3}\right)+\beta_{2} x_{2}\left(g x_{1} x_{3}-k x_{2}+u_{o 1}\right) \\
& +\beta_{3} x_{3}\left(l x_{2}+q x_{4} x_{5}-m x_{3}+u_{o 2}\right)+\beta_{4} x_{4}\left(s_{T}+s x_{2} x_{4}-\mu_{T} x_{4}+u_{o 3}\right) \\
& +\beta_{5} x_{5}\left(s_{B}-x_{5} x_{4}-\mu_{B} x_{5}\right)
\end{aligned}
$$

the control inputs are Substituting the control inputs (39) into (42), then

$$
\begin{aligned}
\dot{L}_{c}= & \beta_{1} a x_{1}-\beta_{1} c x_{1}^{2}+\beta_{1}(b+k) x_{1} x_{2}-\beta_{2} k x_{2}^{2}+\beta_{2} \delta_{1} x_{2} \\
& -\beta_{3} m x_{3}^{2}+\beta_{3} \delta_{2} x_{3}-\beta_{4} \mu_{T} x_{4}^{2}+\beta_{4} s_{T} x_{4}+\beta_{4} \delta_{3} x_{4} \\
& +\beta_{5} s_{B} x_{5}-\beta_{5} \mu_{B} x_{5}^{2}-\beta_{1} g x_{1}^{2} x_{3}-\beta_{5} x_{5}^{2} x_{4}
\end{aligned}
$$

Hence,completing the quadratic form for variables $x_{3}, x_{4}$, and $x_{5}$ gives that $\dot{L}_{c}$ is as follows:

$$
\left\{\begin{array}{l}
\beta_{3} \delta_{2} x_{3}-\beta_{3} m x_{3}^{2}=-\beta_{3} m\left(x_{3}-\frac{\delta_{2}}{2 m}\right)^{2}+\beta_{3} \frac{\delta_{2}^{2}}{4 m} \\
\beta_{4}\left(s_{T}+\delta_{3}\right) x_{4}-\beta_{4} \mu_{T} x_{4}^{2}=-\beta_{4} \mu_{T}\left(x_{4}-\frac{s_{T}+\delta_{3}}{2 \mu_{T}}\right)^{2}+\beta_{4} \frac{\left(s_{T}+\delta_{3}\right)^{2}}{4 \mu_{T}} \\
\beta_{5} s_{B} x_{5}-\beta_{5} \mu_{B} x_{5}^{2}=-\beta_{5} \mu_{B}\left(x_{5}-\frac{S_{B}}{2 \mu_{B}}\right)^{2}+\beta_{5} \frac{S_{B}^{2}}{4 \mu_{B}}
\end{array}\right.
$$



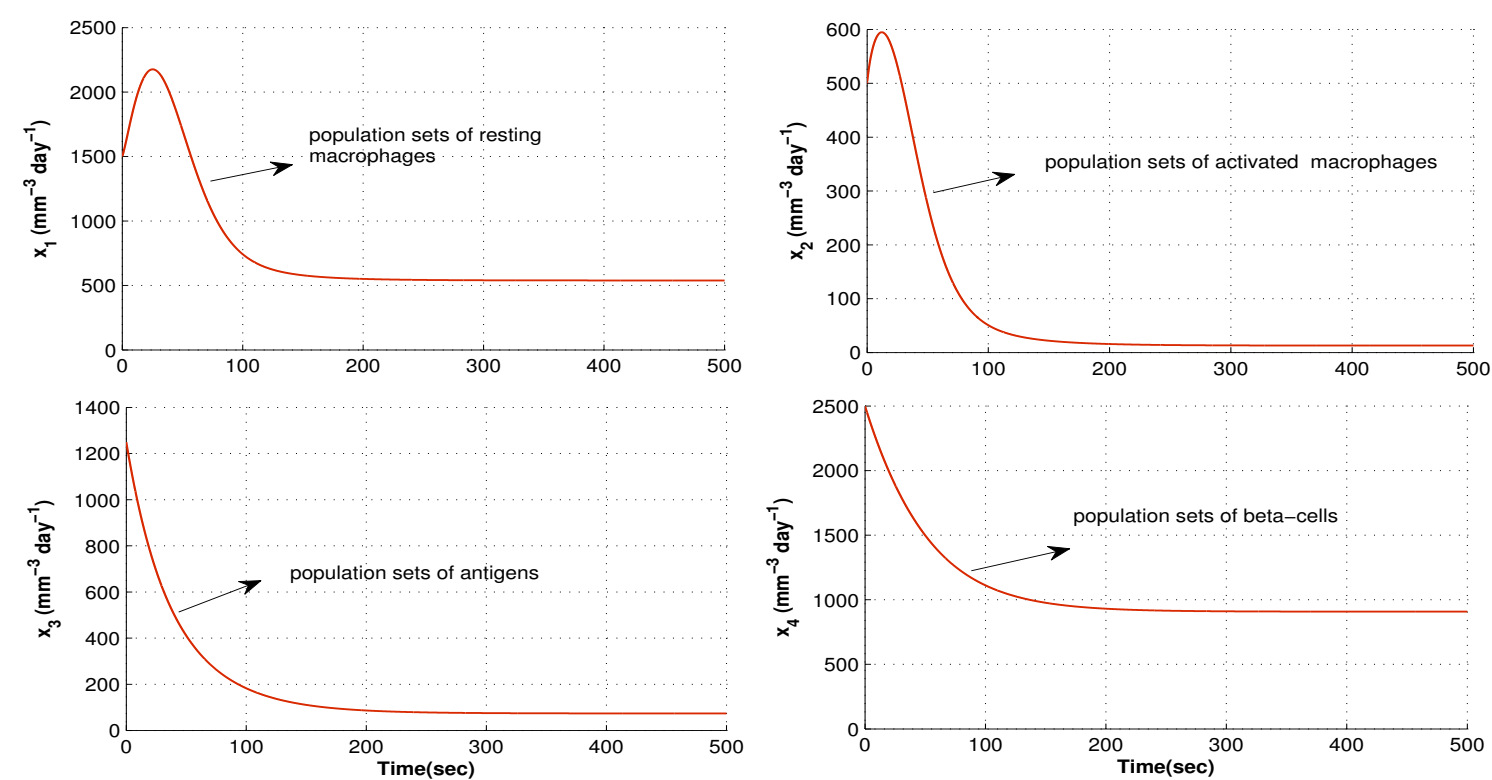

Fig. 4. Upper bound for all cell population sets by the bounded positive invariant domain (BPID) set $K_{1}$, considering the invariant plane $x_{4}(t)=0$.

Now, completing the quadratic form for the positive terms $\left(\beta_{1}(b+k) x_{1} x_{2}-\beta_{2} k x_{2}^{2}+\beta_{2} \delta_{1} x_{2}\right)$ that contain $x_{1}$ and $x_{2}$ factorizing the common term $\beta_{2}$ as:

$$
\begin{aligned}
\beta_{2}\left(\frac{\beta_{1}(b+k) x_{1}}{\beta_{2}}+\delta_{1}\right) x_{2}-\beta_{2} k x_{2}^{2}= & -\beta_{2} k\left(x_{2}-\frac{\left(\frac{\beta_{1}(b+k) x_{1}}{\beta_{2}}+\delta_{1}\right)}{2 k}\right)^{2} \\
& +\frac{\beta_{1}^{2}(b+k)^{2} x_{1}^{2}}{4 \beta_{2} k}+\frac{\beta_{2} \delta_{1}^{2}}{4 k}+\frac{\beta_{1} \delta_{1}(b+k) x_{1}}{2 k}
\end{aligned}
$$

using (44) and (45) into (43), the derivative of Lyapunov function can be rewritten as follows:

$$
\begin{aligned}
\dot{L}_{c}= & -\beta_{2} k\left(x_{2}-\frac{\left(\frac{\beta_{1}(b+k) x_{1}}{\beta_{2}}+\delta_{1}\right)}{2 k}\right)^{2}+\frac{\beta_{1}^{2}(b+k)^{2} x_{1}^{2}}{4 \beta_{2} k}+\frac{\beta_{2} \delta_{1}^{2}}{4 k}+\frac{\beta_{1} \delta_{1}(b+k) x_{1}}{2 k} \\
& -\beta_{3} m\left(x_{3}-\frac{\delta_{2}}{2 m}\right)^{2} \beta_{3} \frac{\delta_{2}^{2}}{4 m}-\beta_{4} \mu_{T}\left(x_{4}-\frac{s_{T}+\delta_{3}}{2 \mu_{T}}\right)^{2}+\beta_{4} \frac{\left(s_{T}+\delta_{3}\right)^{2}}{4 \mu_{T}} \\
& -\beta_{5} \mu_{B}\left(x_{5}-\frac{S_{B}}{2 \mu_{B}}\right)^{2}+\beta_{5} \frac{S_{B}^{2}}{4 \mu_{B}}+\beta_{1} a x_{1}-\beta_{1} c x_{1}^{2}-\left(\beta_{1} g x_{1}^{2} x_{3}+\beta_{5} x_{5}^{2} x_{4}\right)
\end{aligned}
$$

and completing the quadratic form for other terms

$$
\beta_{1} l_{1} x_{1}-\beta_{1} l_{2} x_{1}^{2}=-\beta_{1} l_{2}\left(x_{1}-\frac{l_{1}}{2 l_{2}}\right)^{2}+\beta_{1} \frac{l_{1}^{2}}{4 l_{2}}
$$

So, the equation (46) is defined as:

$$
\begin{aligned}
\dot{L}_{c}= & -\beta_{2} k\left(x_{2}-\frac{\left(\frac{\beta_{1}(b+k) x_{1}}{\beta_{2}}+\delta_{1}\right)}{2 k}\right)^{2}-\beta_{3} m\left(x_{3}-\frac{\delta_{2}}{2 m}\right)^{2}-\beta_{4} \mu_{T}\left(x_{4}-\frac{s_{T}+\delta_{3}}{2 \mu_{T}}\right)^{2}-\beta_{5} \mu_{B}\left(x_{5}-\frac{S_{B}}{2 \mu_{B}}\right)^{2} \\
& -\beta_{1} l_{2}\left(x_{1}-\frac{l_{1}}{2 l_{2}}\right)^{2}-\left(\beta_{1} g x_{1}^{2} x_{3}+\beta_{5} x_{5}^{2} x_{4}\right)+\beta_{1} \frac{l_{1}^{2}}{4 l_{2}}+\beta_{5} \frac{S_{B}^{2}}{4 \mu_{B}}+\beta_{4} \frac{\left(s_{T}+\delta_{3}\right)^{2}}{4 \mu_{T}}+\beta_{3} \frac{\delta_{2}^{2}}{4 m}+\frac{\beta_{2} \delta_{1}^{2}}{4 k}
\end{aligned}
$$


with

$$
l_{1}=a+\frac{\beta_{1} \delta_{1}(b+k)}{2 k} \quad, \quad l_{2}=c-\frac{\beta_{1}^{2}(b+k)^{2}}{4 \beta_{2} k}
$$

where the following condition for $\beta_{2}$ must be satisfied to guarantee the positiveness of $h$ :

$$
\beta_{2}>\frac{\beta_{1}(b+k)^{2}}{4 c k}
$$

Hence, since all variables present nonlinear dynamics in the positive orthant due to their biological implications, and as the analysis demonstrates in the previous section, equation (47) satisfies Lyapunov asymptotic stability if the following inequality is also satisfied:

$$
\begin{array}{r}
+\beta_{2} k\left(x_{2}-\frac{\left(\frac{\beta_{1}(b+k) x_{1}}{\beta_{2}}+\delta_{1}\right)}{2 k}\right)^{2}+\beta_{3} m\left(x_{3}-\frac{\delta_{2}}{2 m}\right)^{2}+\beta_{4} \mu_{T}\left(x_{4}-\frac{s_{T}+\delta_{3}}{2 \mu_{T}}\right)^{2}+\beta_{5} \mu_{B}\left(x_{5}-\frac{S_{B}}{2 \mu_{B}}\right)^{2} \\
+\beta_{1} l_{2}\left(x_{1}-\frac{l_{1}}{2 l_{2}}\right)^{2}+\left(\beta_{1} g x_{1}^{2} x_{3}+\beta_{5} x_{5}^{2} x_{4}\right)>S_{3}
\end{array}
$$

where

$$
S_{3}=\beta_{1} \frac{l_{1}^{2}}{4 l_{2}}+\beta_{5} \frac{S_{B}^{2}}{4 \mu_{B}}+\beta_{4} \frac{\left(s_{T}+\delta_{3}\right)^{2}}{4 \mu_{T}}+\beta_{3} \frac{\delta_{2}^{2}}{4 m}+\frac{\beta_{2} \delta_{1}^{2}}{4 k}
$$

The proposed control inputs are biologically sound. Moreover, a physical implementation will be still a future challenge because it is hard to modify cell populations such as resting macrophages, since they are produced naturally by the immune system. However, It is obvious that the system with the proposed control inputs is stable. Therefore, applying the proposed controller to patients can be very helpful and effective.

\section{Numerical Simulation}

In this section, the numerical simulation is presented. The parameter values of the model for the simulation setting are given in Table 1.

Fig. 4 shows the bounded positive invariant domain construct with the invariant plane K1. As can be seen, when there is no set of T-cells concentration into the system, regardless of the initial populations of the cells that remain inside it, they will tend to their equilibrium level, which means a stable condition of the disease where there is no progression. Therefore, it can be concluded that the population of $\beta$-cells in the pancreas is optimal.

In this case, this simulation is considered valuable due to the feasibility of proposing at least one control input for the system (8) that could help to ensure a stable population of T-cells, or at least prevent their indiscriminate spread. Moreover, the invariant plane of $x_{4}=0$ implies that there is no immunological response. Therefore, all cell populations tend to their optimal concentrations.

In Fig. 5(a-e), the responses of Type-1 Diabetes under the adaptive observer-based controller are shown. The upper bound domain of the localizing set for both activated and resting macrophages is shown in Fig. 5(a) and Fig. 5(b). Also, the upper bound domain of the localizing for antigen cells concentration is displayed in Fig. 5(c) which the variables associated with activated macrophages have immediate response once the $\beta$-cells are presented as antigen. It is a natural response, since they influence the lymphocyte cells by activating the autolytic T-cells. Fig. 5(d) presents the upper and lower bounds for the T-cell population. As can be seen, it is important to highlight that exists a minimum level of a cell population that has a direct impact on triggering cytotoxic T-cells. Finally, the upper bound for the b-cell population resulting is demonstrated in Fig. 5(e). Therefore, this analysis exposes the complex interaction between these cell populations, demonstrating that when there are no activated macrophages that label a population set of $\beta$-cells as antigens, there is no autolytic response required.

Furthermore, Fig. 6 and Fig. 7 just like Fig. 5, show the model response with the proposed controller with different initial conditions. According to these figures, it can be seen that if there is no control action, 
Table 1. Parameter descriptions, values, and units

\begin{tabular}{llll}
\hline Parameter & Definition & Value & Units \\
\hline \hline $\mathrm{a}$ & Macrophage supply & 50 & $\mathrm{~mm}^{-3} \mathrm{day}^{-1}$ \\
$\mathrm{~b}$ & Macrophage induced supply & 0.3 & $\mathrm{day}^{-1}$ \\
$\mathrm{c}$ & Macrophage death rate & 0.1 & $\mathrm{~mm}^{-3} \mathrm{day}^{-1}$ \\
$\mathrm{~g}$ & Rate of antigen uptake & $65 \times 10^{-6}$ & $\mathrm{day}^{-1}$ \\
$\mathrm{k}$ & Macrophage deactivation & 0.2 & $\mathrm{day}^{-1}$ \\
$\mathrm{l}$ & Induced b-cell damage & $250 \times 10^{-6}$ & $\mathrm{day}^{-1}$ \\
$\mathrm{~m}$ & Decay rate of b-cell proteins & 0.025 & $\mathrm{~mm}^{-3} \mathrm{day}^{-1}$ \\
$\mathrm{q}$ & Damage of autolytic cells on $\beta$-cells & $2 \times 10^{-6}$ & $\mathrm{~mm}^{-3} \mathrm{day}^{-1}$ \\
$s_{T}$ & Supply of autolytic cells & 20 & $\mathrm{day}^{-1}$ \\
$\mathrm{~s}$ & Proliferation of autolytic T-cells & $2 \times 10^{-5}$ & $\mathrm{day}^{-1}$ \\
$\mu_{T}$ & Death rate of T-cells & 0.02 & $\mathrm{day}^{-1}$ \\
$s_{B}$ & Supply of $\beta$-cells & 20 & $\mathrm{~mm}^{-3} \mathrm{day}^{-1}$ \\
$\mu_{B}$ & Death rate of $\beta$-cells & 0.02 & $\mathrm{~mm}^{-3} \mathrm{day}^{-1}$ \\
\hline
\end{tabular}

cell populations will not reach their state of equilibrium and the system is susceptible to parametric variation that could generate diabetes. Therefore, this leads to the development of a mathematical proposal supported by the nonlinear neural adaptive observer-based controller theory to treat disease.

A mathematical analysis of a nonlinear model aimed at understanding the complex relation between $\beta$-cells with the immunological response through autolytic $T$-cells and macrophages demand a deeper understanding of biological concepts related to Type-1 Diabetes. According to the literature, in diabetes there is an inverse correlation between b-cells and autolytic T-cells, implying that when the autolytic Tcell population increases, the $\beta$-cell population decreases, activating a diabetic condition. At this point, we note that in a steady-state condition, cell populations never reach their state of equilibrium (see Fig. 5 ), some of them even tend to their upper or lower bounds, such as the case of resting macrophages, activated macrophages, and autolytic T-cells, while an increase of $\beta$-cells is due to T-cell population decreases. Nevertheless, as can be seen in Fig. 5(c) and Fig. 5(d), the $\beta$-cells will not be greater in number than the autolytic T-cells because $\beta$-cells upper bound becomes the autolytic T-cells lower bound; at most, these populations would be equal. Therefore, it is vital to notice that autolytic T-cells cannot be zero, in any case, making it so that a diabetic condition could appear at any time. The above emphasizes the importance of a closed-loop control system able to compensate for the proliferation of autolytic T-cells against the death rate of b-cells. In this study, we propose a closed-loop control system and have proven that the control inputs, in accordance with the mathematical analysis, can keep cell populations at a stable level towards diabetes control in early diagnostics.

\section{Conclusion}

In this paper, a new adaptive neural observer-based controller for a class of nonlinear diabetes models was presented. Type 1 diabetes is a growing health problem worldwide because they have a deficiency in insulin production. The proposed strategy was presented as a solution that guarantees practical tracking of a desired glucose concentration. Moreover, the feed-forward neural network was used to estimate the internal state of diabetes that was a set of inputs onto a set of suitable outputs. The network under online learning is one of the artificial intelligence methods which was trained online that the learning method was a simple backpropagation algorithm. The promising performance of the mentioned controller was depicted in simulation results. A physical implementation will be future work.

\section{References}

[1] K. Eskaf, O. Badawi, and T. Ritchings, "Predicting Blood Glucose Levels in Diabetics Using Feature Extraction and Artificial Neural Networks," in Proc. of Int. Conf. on Information and Communication Technologies: From Theory to Applications, Damascus, Syria, pp. 1-6, 2008. https://doi.org/10. 

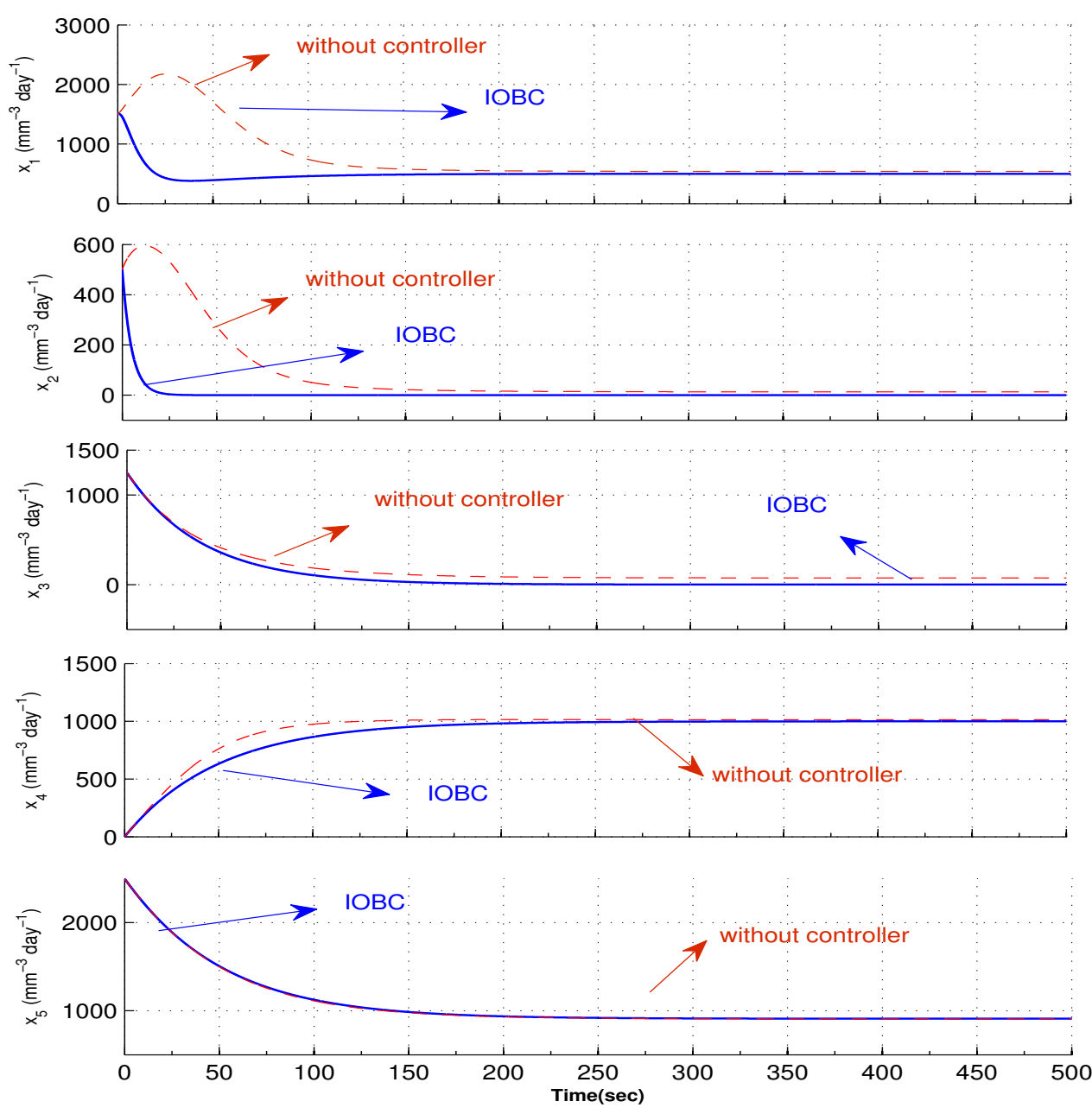

Fig. 5. Upper and lower bounds for each cell populations. (a) Maximum concentration of resting macrophage cells. (b) Maximum concentration of activated macrophage cells. (c) Maximum concentration of antigen cells. (d) Minimum and maximum concentrations of T-cells. (e) Maximum concentration of b-cells.

\section{9/ICTTA.2008.4529940}

[2] O. Vega-Hernandez, D. U. Campos-Delgado and D. R. Espinoza-Trejo, "Increasing security in an artificial pancreas: diagnosis of actuator faults," in Proc. of Pan-American Health Care Exchanges, 2009, pp. 137-142. https://doi.org/10.1109/PAHCE.2009.5158384

[3] V. R. Kondepati and H. M. Heise, "Recent progress in analytical instrumentation for glycemic control in diabetic and critically ill patients," Anal Bional Chem, vol.388, pp. 545-563, 2007. https://doi.org/ 10.1007/s00216-007-1229-8

[4] S. Thomson, D. Beaven, M. Jamieson, S. Snively, A. Howl and A. Christophersen, "Type II diabetes: Managing for better health outcomes," in Price Water House Coopers Report, Diabetes New Zealand Inc, 2001.

[5] B. Topp, K. Promislow and G. De Vries, "A model of-cell mass, insulin and glucose kinetics," Pathways to diabetes. J. Theor. Bio, pp. 605-619, 2000. https://doi.org/10.1006/jtbi.2000.2150

[6] S. M. Lynch and B. W. Bequette, "Estimation-based model predictive control of blood glucose in type I diabetics: A simulation study," Proceedings of the IEEE 27th Annual Northeast Bioengineering Conference, Storrs, CT, USA, pp. 79-80, 2001. 

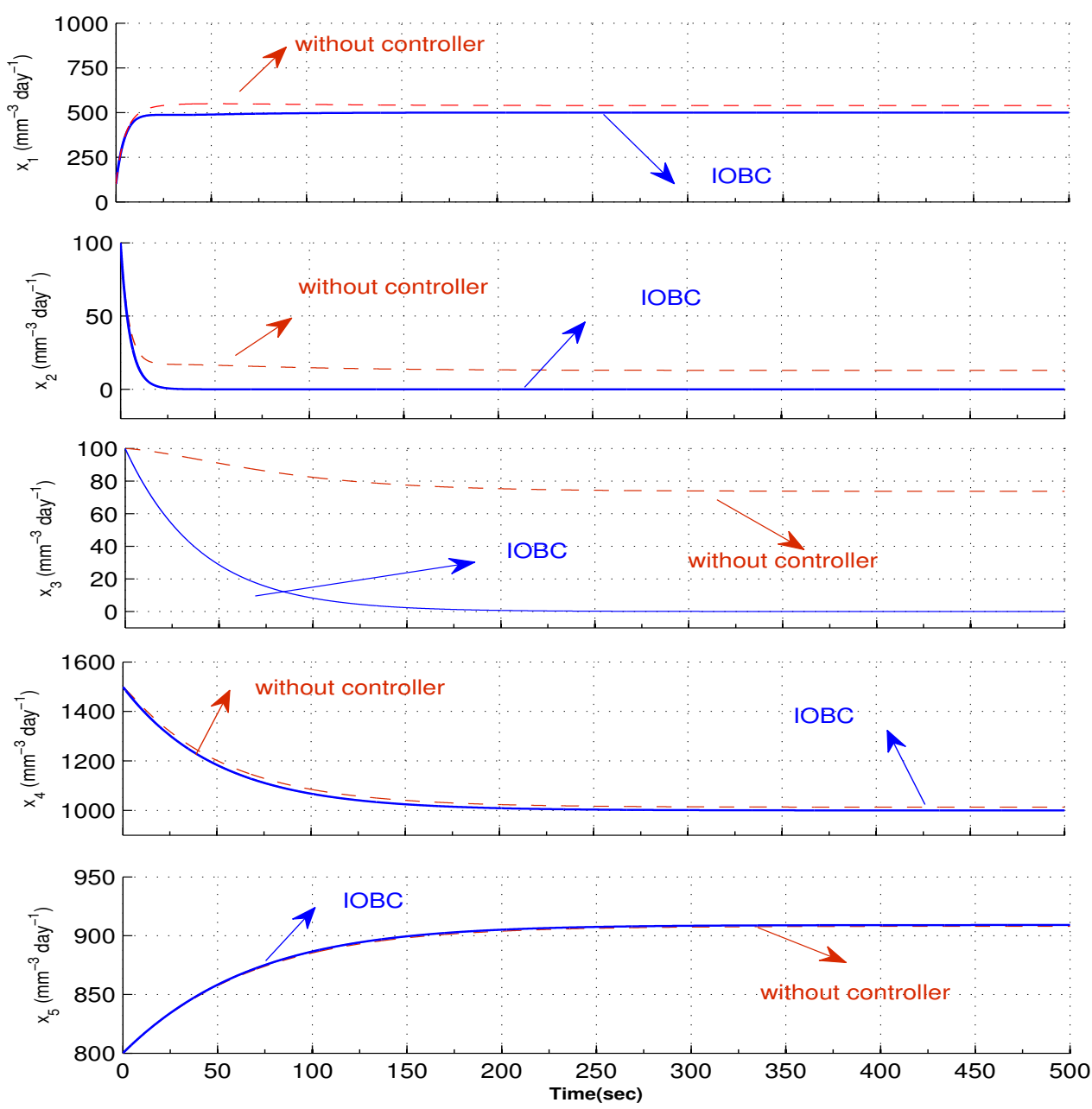

Fig. 6. Upper and lower bounds for each cell populations. (a) Maximum concentration of resting macrophage cells. (b) Maximum concentration of activated macrophage cells. (c) Maximum concentration of antigen cells. (d) Minimum and maximum concentrations of T-cells. (e) Maximum concentration of b-cells.

[7] G. P. Forlenza and M. Rewers, "The epidemic of type 1 diabetes: what is it telling us?" Curr. Opin. Endocrinol. Diabetes Obes, vol.18, pp. 248-251, 2011. https://doi.org/10.1097/MED. 0b013e32834872ce

[8] P. Saeedi, I. Petersohn, P. Salpea, B. Malanda, S. Karuranga, N. Unwin, S. Colagiuri, L. Guariguata, A. A. Motala, K. Ogurtsova, J. E. Shaw, D. Bright, R. Williams, "Global and Regional Diabetes Prevalence Estimates for 2019 and Projections for 2030 and 2045," Diabetes Research and Clinical Practice, vol. 157, p. 107843, 2019. https://doi.org/10.1016/j.diabres.2019.107843

[9] K. Saarela, J. Tuomilehto, R. Sund, I. Keskimaki, S. Hartikainen, E. Pukkala, "Cancer incidence among Finnish people with type 2 diabetes during 1989-2014," Eur. J. Epidemiol, vol. 34, pp. 259-265, 2019. https://doi.org/10.1007/s10654-018-0438-0

[10] B. Zhou, Y. Lu, K. Hajifathalian, J. Bentham, M. Di Cesare, G. Danaei, H. Bixby, M. Cowan, M. Ali, C. Taddei, et. al. "Worldwide trends in diabetes since 1980: A pooled analysis of 751 population-based studies with 44 million participants," The Lancet, vol. 387, no. 10027, pp. 1513-1530, 2016. https: //doi.org/10.1016/S0140-6736(16)00618-8

[11] J. L. Harding, M. E. Pavkov, D. J. Magliano, J. E. Shaw, E. W. Gregg, "Global trends in diabetes complications: A review of current evidence," Diabetologia, vol. 62, pp. 3-16, 2019. https://doi.org/10.1007/ s00125-018-4711-2 

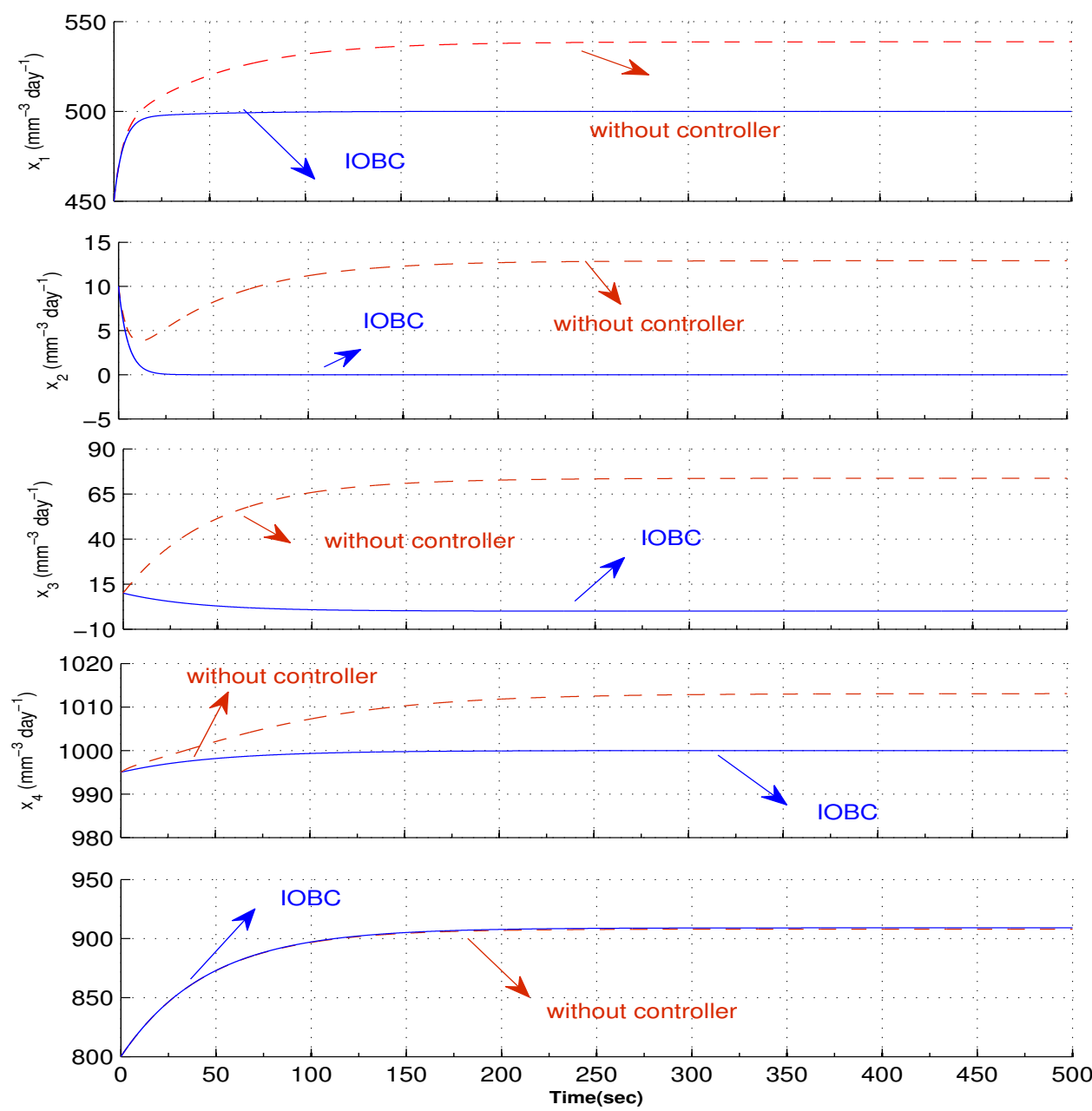

Fig. 7. Upper and lower bounds for each cell populations. (a) Maximum concentration of resting macrophage cells. (b) Maximum concentration of activated macrophage cells. (c) Maximum concentration of antigen cells. (d) Minimum and maximum concentrations of T-cells. (e) Maximum concentration of b-cells.

[12] N. H. Cho, J. E. Shaw, S. Karuranga, Y. Huang, J. D. da R. Fernandes, A. W. Ohlrogge and B. Malanda, "IDF Diabetes Atlas: Global estimates of diabetes prevalence for 2017 and projections for 2045," Diabetes Res. Clin. Pract, vol. 138, pp. 271-281, 2018. https://doi.org/10.1016/j.diabres.2018.02.023

[13] R. Ghasemi and M. R. R. Khoygani, "Designing Intelligent Adaptive Controller for Nonlinear Pendulum Dynamical System," International Journal of Energy and Power Engineering, vol. 8, no. 11, pp. 1755-1759, 2014. https://citeseerx.ist.psu.edu/viewdoc/download?doi=10.1.1.667.4732\& rep=rep1\&type=pdf

[14] M. R. R. Khoygani, R. Ghasemi and D Sanaei, "Designing Controller for Joined Dynamic Nonlinear PEMFC and Buck Converter System," International Journal of Power Electronics and Drive System, vol. 4, no. 4, pp. 137-145, 2014. https://doi.org/10.11591/ijpeds.v4i2.5195

[15] M. R. R. Khoygani, R. Ghasemi and D. Sanaei, "Design Controller for a Class of Nonlinear Pendulum Dynamical System," IAES International Journal of Artificial Intelligence, vol. 2, no. 4, pp. 159-168, 2013. https://doi.org/10.11591/ij-ai.v2i4.4164

[16] M. R. Rahimi, R. Ghasemi and D. Sanaei, "Designing Discrete Time Optimal Controller for Double Inverted Pendulum System," International Journal on Numerical and Analytical Methods in Engineering, vol. 1, no. 1, 2013. https://www.praiseworthyprize.org/jsm/index.php?journal=irena\&page= article\&op=view\&path\%5B\%5D=10928 
[17] M. R. R. Khoygani, R. Ghasemi, P. Ghayoomi "Robust Observer-based Control of Nonlinear MultiOmnidirectional Wheeled Robot Systems via High Order Sliding-mode Consensus Protocol," International Journal of Automation and Computing, vol. 18, pp. 787-801, 2021. https://doi.org/10. 1007/s11633-020-1254-z

[18] M. R. R. Khoygani, R. Ghasemi, "Neural estimation using a stable discrete-time MLP observer for a class of discrete-time uncertain nonlinear systems," Nonlinear Dynamics, vol. 84, no. 4, pp. 25172533, 2016. https://doi.org/10.1007/s11071-016-2662-z

[19] M. R. R. Khoygani, R. Ghasemi and Ahmad Reza Vali, "Intelligent nonlinear observer design for a class of nonlinear discrete-time flexible joint robot," Intelligent Service Robotics, vol. 8, no. 1, pp. 45-56, 2015. https://doi.org/10.1007/s11370-014-0162-x

[20] M. Bakhti, A. Bottcher and H. Lickert, "Modelling the endocrine pancreas in health and disease," Nat. Rev. Endocrinol, vol. 15, pp. 155-171, 2019. https://doi.org/10.1038/s41574-018-0132-z

[21] Li, C. and R. Hu, "PID Control Based on BP Neural Network for the Regulation of Blood Glucose Level in Diabetes," in Proceeding of the 7th IEEE International Conference on Bioinformatics and Bioengineering, Boston, pp. 1168-1172, 2007. https://doi.org/10.1109/BIBE.2007.4375709

[22] F. Chee, T. L. Fernando, A. V. Savkin, and V. van Heeden, "Expert PID control system for blood glucose control in critically ill patients," IEEE Transactions on Information Technology in Biomedicine, vol. 7, no. 4, pp. 419-425, 2003. https://doi.org/10.1109/TITB.2003.821326

[23] E. Salzsieder, G. Albrecht, U. Fischer, E.-J. Freyse, "Kinetic modeling of the glucoregulatory system to improve insulin therapy," IEEE Transactions on Biomedical Engineering, vol. BME-32, no. 10, pp. 846-855, 1985. https://doi.org/10.1109/TBME.1985.325500

[24] R. S. Parker, F. J. Doyle and N. A. Peppas, "A model-based algorithm for blood glucose control in type I diabetic patients," IEEE Transactions on Biomedical Engineering, vol. 46, no. 2, pp.148-157, 1999. https://doi.org/10.1109/10.740877

[25] S. M. Lynch and B. W. Bequette, "Model predictive control of blood glucose in type I diabetes using subcutaneous glucose measurements," in Proceedings of the American Control Conference, 2002, pp. 4039-4040. https://doi.org/10.1109/ACC.2002.1024561

[26] C. Li and R. Hu, "PID Control Based on BP Neural Network for the Regulation of Blood Glucose Level in Diabetes," in the 7th IEEE International Conference on Bioinformatics and Bioengineering, Boston, pp. 1168-1172, 2007. https://doi.org/10.1109/BIBE.2007.4375709

[27] K. Beyki, M. D. Javan, S. G. Kambiz and M. M. Neshati, "An intelligent approach for optimal regulation of blood glucose level," In 17th Iranian Conference of Biomedical Engineering, 2010, pp. 1-5. https: //doi.org/10.1109/ICBME.2010.5704984

[28] S. Yasini, M. B. Naghibi-Sistani and A. Karimpour, "Active insulin infusion using fuzzy-based closedloop control," in 3rd International Conference on Intelligent System and Knowledge Engineering, Mashhad, Iran, pp. 429-434, 2008. https://doi.org/10.1109/ISKE.2008.4730968

[29] D. N. M. Abadi, A. Alfi and M. Siahi, "An Improved Fuzzy PI Controller for Type 1 Diabetes," Research Journal of Applied Sciences, Engineering and Technology, vol. 4, no. 21, pp. 4417-4422, 2012. https://www.airitilibrary.com/Publication/alDetailedMesh?docid= 20407467-201211-201512080011-201512080011-4417-4422

[30] G. Magombedze, P. Nduru, C. P. Bhunu and S. Mushayabasa, "Mathematical modelling of immune regulation of type 1 diabetes," Biosystems, vol. 102, no. 2-3, pp. 88-98, 2010. https://doi.org/10. 1016/j.biosystems.2010.07.018

[31] O. D.Sanchez, A. Y. Alanis, E. R. Velázquez and R. V. Murillo, "Neural identification of Type 1 Diabetes Mellitus for care and forecasting of risk events," Expert Systems with Applications, vol. 183, p. 115367, 2021. https://doi.org/10.1016/j.eswa.2021.115367

[32] T. Padmapritha and B. Subathra, "Smart Artificial Pancreas with Diet Recommender System for Elderly Diabetes," IFAC-PapersOnLine, Vol.53, Issue 2, pp. 16366-16371, 2020. https://doi.org/10. 1016/j.ifacol.2020.12.670

[33] A. Ma'arif, A. I. Cahyadi, S. Herdjunanto, and O. Wahyunggoro, "Tracking Control of High Order Input Reference Using Integrals State Feedback and Coefficient Diagram Method Tuning," IEEE Access, vol. 8, pp. 182731-182741, 2020. https://doi.org/10.1109/ACCESS.2020.3029115 
[34] D. S. Febriyan and R. D. Puriyanto, "Implementation of DC Motor PID Control On Conveyor for Separating Potato Seeds by Weight," International Journal of Robotics and Control Systems, vol. 1, no. 1, pp. 15-26, 2021. https://doi.org/10.31763/ijrcs.v1i1.221 